\title{
Parametrizing the Net Maternity Function
}

\author{
Jonathan D. H. Smith ${ }^{1,}{ }^{*}$, Chaoliang Zhang ${ }^{1}$
}

\begin{abstract}
The net maternity function represents the chance of a newborn female surviving to a certain age class, multiplied by the average number of female babies she is likely to bear while sojourning in that age class. Although the net maternity function does not lend itself well to modeling in terms of conventional time, it may be parametrized effectively as a function of the logarithm of a mother's age. Choice of this independent variable leads to absolute dimensionless quantities that do not have to be rescaled when the units of time are changed. The parametrized model provides a close fit to the real net maternity function for stable populations, while divergences between the model and actual function may serve as a diagnostic for new features within a population. Reduction of the specification of the net maternity function to a small number of parameters enables those parameters to be tracked in long-term historical studies, and also serves as the basis of techniques for predicting future states of a population. Among the parameters, a new logarithmic generation time emerges as an important population variable.
\end{abstract}

Keywords: net maternity function, generation time, organic time, physiological time, lognormal distribution

\section{Introduction}

In a given human population, the discrete net maternity function is a function of the age of a mother, describing the chance of a newborn female surviving to a certain age class (say from 20 to 25 years), multiplied by the average number of female babies she is likely to bear while sojourning in that age class. Modeling and parametrization of the net maternity function has always posed a challenge, since it does not appear to fit any of the usual distribution patterns [6].

In this paper, it is argued that the logarithm of a mother's age $a$ is the most appropriate reference variable for the net maternity function ( 3.1). With this choice, the key quantities become dimensionless, and thus have an absolute numerical significance independent of any specific choice of units (3.2). The continuous version $\varphi(a)$ of the net maternity function may then be parametrized (3.4), with a high degree of accuracy in stable populations ( 3.5$)$, as

$$
\varphi(a)=\frac{R_{-1}}{u \overline{2 \pi}} \exp \left[-\frac{1}{2 u^{2}}\left(\log \frac{a}{t}\right)^{2}\right]
$$

in terms of just three parameters ( $3.2-3.3)$ : a renormalization constant $R_{-1}$ (per annum), a logarithmic generation time $t$ (in years), and $u$ as a dimensionless deviation. The logarithmic generation time emerges as an important population variable in its own right, and not just a proxy for the classical generation time ( 3.3.1).

\footnotetext{
${ }^{1}$ Department of Mathematics, Iowa State University, Ames, IA 50011

${ }^{*}$ Correspondence: jdhsmith@iastate.edu
} 
Section 2 reviews the standard features of the net maternity function that are required. Section 3 then presents the new parametric modeling approach, based on the logarithmic time. Section 4 examines three interrelated applications of the new model: diagnostics ( 4.1, 4.2.1), prediction (4.2), and long-term tracking ( 4.3-4.4). A remarkable conservation principle that emerges experimentally from such long-term tracking of the Swedish population over the period from 1780 to 1925 ( 4.4.4) enables one to eliminate the deviation $u$ from the parametrization (1), reducing to a dependence only on the renormalization constant and the logarithmic generation time.

\section{The net maternity function}

This section provides a quick review of the net maternity function as it is known from the literature, in order to establish a background for the new approach presented in the paper.

\subsection{The Leslie matrix}

The development of a human population over time has traditionally been modeled using fiveyear age classes and the Leslie or projection matrix, although other essentially equivalent formulations, e.g., using continuous variables, are also used $[6,7]$. The Leslie matrix is the incidence matrix

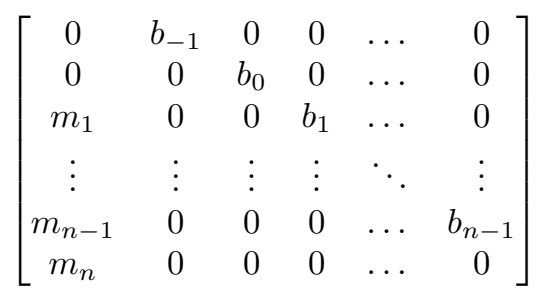

of the weighted graph displayed in Figure 1 (truncating the infertile age classes) ${ }^{1}$. The

$$
\begin{aligned}
& \text { 1: }<5 \text { years } \\
& \text { 0: 5-9 years } \\
& \text { 1: } 10-14 \text { years } \\
& \text { 2: } 15-19 \text { years } \\
& \text { 3: 20-24 years }
\end{aligned}
$$

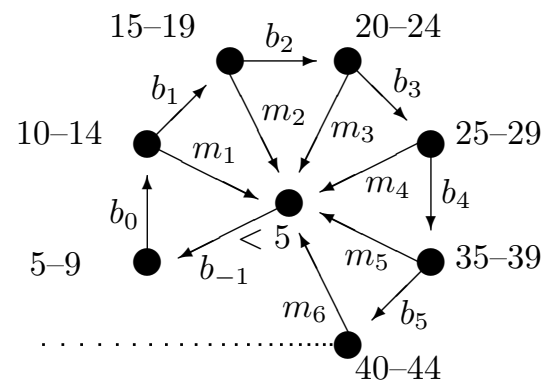

Figure 1: Age classes in a female population. The weight $b_{i}$ is the rate of survival from the $i$-th age class to the $(i+1)$-st. The weight $m_{i}$ is the expected number of girls born to a mother during her time in the $i$-th age class.

graph depicts the various demographically relevant age classes within a population of human females, based on intervals of 5 years. In the center are the newborns, less than 5 years old. The path from the center leading round the outside of the picture describes the passage of a female through the various age classes. The arrows on this path are weighted by rates of survival $b{ }_{1}, b_{0}, b_{1}, \ldots$ from each age class to the next, $b_{i}$ being the proportion of females from age class $i$ who survive and move into age class $i+1$. (In terms of the quantities computed from census data and tabulated in $[8,9], b_{i}={ }_{5} L_{x+5} /{ }_{5} L_{x}$, where $x$ denotes the

\footnotetext{
${ }^{1}$ If one works with column vectors and matrices multiplying from the left, the Leslie matrix will appear as the transpose of (2).
} 
age at which the age class $i$ begins.) The arrows leading in towards the center from each reproductive age class indicate the daughters born to a female during her time in that class. These arrows are weighted by the average number $m_{i}$ of female babies that are born to the mothers in the class.

\subsection{The generation time}

Let $p_{i}$ denote the probability that the mother of a randomly chosen newborn female lies in age class $i$. Although the data for real populations are presented discretely in terms of these age classes, it is often convenient to pass to a continuous age variable $a$ (in years) for some theoretical analyses, and to replace the dimensionless probabilities $p_{i}$ by a probability density $p(a)$. In other words, if the $i$-th age class covers the interval $t_{1} \quad a<t_{2}$, one has

$$
p_{i}=\int_{t_{1}}^{t_{2}} p(a) d a .
$$

Using the integral formalism, the generation time (in years) is defined as

$$
T=\int_{0} a p(a) d a
$$

the expected age of the mother of a randomly chosen newborn. In typical populations, the generation time normally ranges between 20 and 35 years.

\subsection{The net maternity function}

In the discrete formalism of the Leslie matrix, the net maternity function at an age class $i$ is given by the dimensionless product

$$
\phi_{i}=b{ }_{1} b_{0} b_{1} \ldots b_{i} \quad{ }_{1} m_{i}
$$

which represents the chance of a newborn female surviving to age class $i$, multiplied by the average number of female babies she is likely to bear while sojourning in that age class. Passing to the continuous age variable $a$ (in years), the net maternity function is described by a density function $\phi(a)$ having the dimensions of inverse time (per annum units). In analogy with (3), one has

$$
\phi_{i}=\int_{t_{1}}^{t_{2}} \phi(a) d a
$$

when the age class $i$ covers the age interval $t_{1} \quad a<t_{2}$. The solid line and points + in Figure 2 illustrate a typical net maternity function, in this case for peninsular Malaysia in 1985. The data are taken from [9].

\subsubsection{Remark}

It is important to note that the functions $p(a)$ and $\phi(a)$, or their "binned" discrete versions $p_{i}$ and $\phi_{i}$, share no direct common relationship in general. Equation (5) gives the discrete net maternity function value $\phi_{i}$ directly from the entries of the Leslie matrix for the current population. On the other hand, the probability value $p_{i}$ depends on the relative number of mothers occupying the $i$-th age class. In many population states, this number may depend on historical or external factors that are not fully reflected in the current Leslie matrix.

\subsection{Moments of the net maternity function}

Since

$$
1=\int_{0} \phi(a) d a
$$




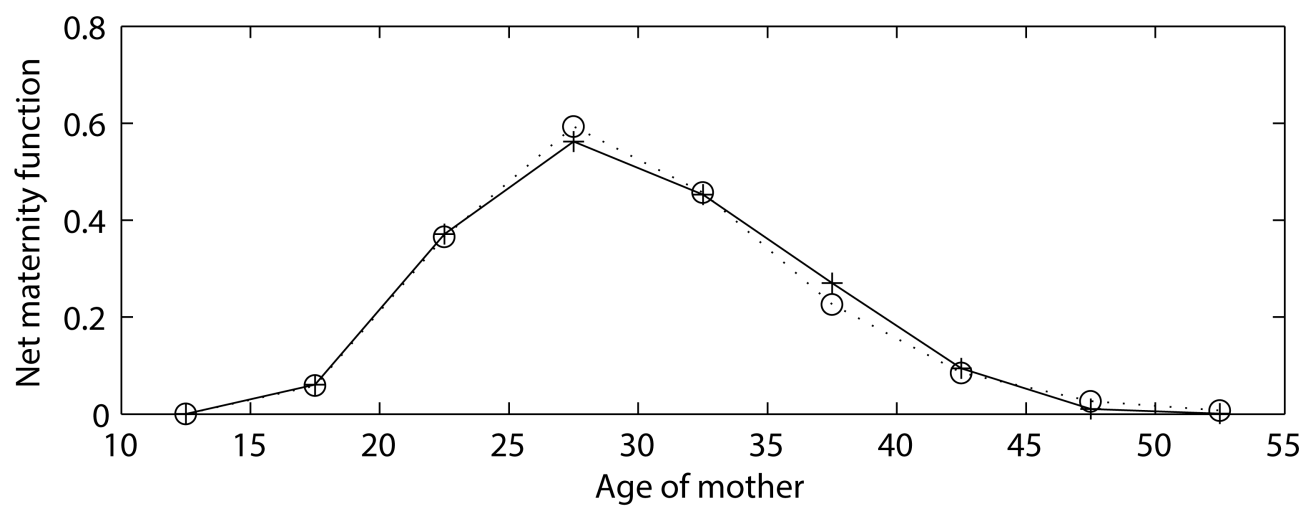

Figure 2: Net maternity functions for Malaysia, 1985. Solid: actual $\phi(a)$. Dotted: the lognormal model $\varphi(a)$.

in general, the net maternity function $\phi(a)$ is not a probability density. Nevertheless, one may define its moments

$$
R_{k}=\int_{0} a^{k} \phi(a) d a
$$

for non-negative integers $k$. In particular, the dimensionless moment $R_{0}$ is known as the reproductive potential or net reproduction rate, "the expected number of girl children by which a girl child now born will be replaced under the observed regime of mortality and fertility" [6, p.140]. Since

$$
\int_{0} \frac{\phi(a)}{R_{0}} d a=1
$$

it is natural to raise the question whether $\phi(a) / R_{0}$ forms a recognizable probability distribution. However, the consensus appears to offer no single satisfactory answer [6, Ch. 6].

\section{Modeling the net maternity function}

This core section outlines the new approach to the net maternity function, leading up to the parametrized model presented in 3.4.1.

\subsection{Logarithmic life-history time}

The starting point for the new approach is the use of a logarithmic time scale for the life history of a mother as the natural independent variable for the net maternity function, in contrast to the usual age $a$ in years that is measured by calendar time or universal time. The practical effectiveness of this choice is demonstrated in 3.5 below. However, there are also three conceptual issues that lead in the same direction, and that are generally relevant for many modeling situations in mathematical biology:

\subsubsection{Physiological time}

Founded on consideration of a wide range of higher organisms, Backman [2] introduced the concept of organic time as a certain linear function of the logarithm of the age of an organism. Although he studied organisms with a spectrum of calendar lifetimes from 3 to $10^{5}$ days, the lifespans of the various organisms became closely comparable in terms of the organic time. Backman and others used this organic time in comparative life cycle studies of different species. Later, the term physiological time gained currency [1, 13]. 


\subsubsection{Thermodynamic time}

Prigogine [12] defined the thermodynamic time $\tau$ of a physical system as the time scale with respect to which the rate of thermodynamic entropy production in the system was constant. Given that this rate $\sigma(t)$ of entropy production generally decreases monotonically with respect to universal time $t$, and tends to zero as $t$ tends to infinity, as at least a first Padé approximation to $\sigma(t)$ one may take the rational function $p_{0} /\left(q_{0}+q_{1} t\right)$. This yields the thermodynamic time $\tau$ as a linear function of the logarithm of the universal time. Subsequently, Prigogine's thermodynamic time has reappeared under the name eigen time [1].

\subsubsection{Dimensional considerations}

The differential of universal time $t$ is $d t$, which again has the dimensions of time. On the other hand, the differential of logarithmic time is $d \log t=d t / t$, a dimensionless reference measure. Thus densities referred to logarithmic time are themselves dimensionless, intrinsic quantities which do not need to be rescaled when units are changed. An additional advantage is seen to accrue when information-theoretic techniques are invoked ( 3.4 .2 below).

\subsection{Renormalization}

By analogy with the specification (7) of the moments, define the renormalization constant

$$
R_{1}=\int_{0} a^{1} \phi(a) d a
$$

in per annum units. The renormalization constant is used to normalize the net maternity function $\phi(a)$ to a dimensionless probability density $\phi(a) / R_{1}$ with respect to the dimensionless logarithmic measure $d a / a$. Thus a formal random variable $X$ lies in the usual time interval $\left[t_{1}, t_{2}\right)$ with probability

$$
\mathrm{P}\left(t_{1} \quad X<t_{2}\right)=\int_{a=t_{1}}^{a=t_{2}} \frac{\phi(a)}{R_{1}} \quad \frac{d a}{a}=\int_{y=\log t_{1}}^{y=\log t_{2}} \frac{\phi\left(e^{y}\right)}{R_{1}} d y
$$

involving the mutually inverse substitutions $a=e^{y}$ and $y=\log a$. In other words, $\phi(a) / R_{1}$ is a density for the logarithmic time $y=\log a$ inherent to the life history of a mother. It is this natural interpretation of $\phi(a) / R_{1}$ which makes it more amenable to parametrization than the awkward per annum density $\phi(a) / R_{0}$ considered in 2.4 .

\subsection{Logarithmic generation time and deviation}

With respect to the density $\phi(a) / R_{1}$, the expected value of the logarithm of the age is

$$
\log t=\int_{0} \frac{\phi(a) \log a}{R_{1} a} d a
$$

in terms of the logarithmic generation time $t$ (units of years). The variance of the logarithm of the age is

$$
u^{2}=\int_{0} \frac{\phi(a)[\log (a / t)]^{2}}{R_{1} a} d a
$$

in terms of the dimensionless, positive (standard) deviation $u$. 


\subsubsection{Comparing classical and logarithmic generation times}

Generally (based on computations with several dozen historic populations from $[8,9]$ ), the logarithmic generation time $t$ will be less than the classical generation time $T$ of (4). Note the values for $t$ and $T$ in the 1970-1985 Malaysian populations displayed in Table 1, for example. In that table, it is also interesting to compare the two generation times for 1970 and 1980. While the classical generation time decreases from 27.83 to 27.75 years, the logarithmic generation time increases from 27.09 to 27.24 years. Thus the logarithmic generation time should be considered as an important independent population variable in its own right, and not merely as a proxy for the classical generation time.

\subsection{The (log)normal distribution}

Suppose that nothing is known about the density $\phi(a) / R_{1}$ except the mean $\log t$ and variance $u^{2}$. Then the appropriate density is normal with respect to logarithmic time. In statistical settings, the normal distribution is usually justified by the Central Limit Theorem, but that justification does not appear to be relevant in the present context. Rather, one observes that amongst all well-behaved non-negative functions $q(a)$, the problem of maximizing the information-theoretic or "Shannon" entropy

$$
\int_{0} q(a) \log q(a) \frac{d a}{a}
$$

subject to the constraints

$$
\log t=\int_{0} q(a) \log a \frac{d a}{a}
$$

and

$$
u^{2}=\int_{0} q(a)[\log (a / t)]^{2} \frac{d a}{a}
$$

corresponding to (9) and (10) respectively, along with the constraint

$$
1=\int_{0} q(a) \frac{d a}{a}
$$

of seeking a probability distribution, has

$$
q(a)=\frac{1}{u \overline{2 \pi}} \exp \left[\frac{1}{2 u^{2}}\left(\log \frac{a}{t}\right)^{2}\right]
$$

as its unique solution [14, p.629]. Note that $q(a) / a$ is a lognormal probability density with respect to the measure $d a$ (compare [3]). A game-theoretical approach, more general than the entropy maximization from certain points of view, is discussed in [4].

\subsubsection{The lognormal model net maternity function}

As a summary of the preceding observations, one may explicitly display the model net maternity function $\varphi(a)$ in terms of the parameters $R_{1}, t, u$ as

$$
\varphi(a)=\frac{R}{u \frac{1}{2 \pi}} \exp \left[\frac{1}{2 u^{2}}\left(\log \frac{a}{t}\right)^{2}\right] .
$$

This is the lognormal model net maternity function. 


\subsubsection{Correctness of the entropy formula}

The general "Shannon" entropy formula

$$
\int p(x) \log p(x) d x
$$

is not well defined when $x$ has a dimension, since the dimensioned density $p(x)$ may not then be used as an argument for the logarithm [11, 15]. However, as argued in 3.1.3 and 3.2, the formula (11) is immune from this issue, due to the use of the logarithmic time that renders the density $q(a)$ dimensionless.

\subsubsection{Correctness of the constraints}

In the constraint (12), and in its prototype (9), arguments $a$ and $t$ with the dimensions of time are explicitly used in logarithm functions. Thus it might appear that the issue raised with the "Shannon" entropy in 3.4.2 would also arise in (12) and (9). However, one may regard (12) and (9) as respective simplified equivalents to the dimensionally correct formulas

$$
\log \frac{t}{T}=\int_{0} q(a) \log (a / T) \frac{d a}{a}
$$

and

$$
\log \frac{t}{T}=\int_{0} \frac{\phi(a) \log (a / T)}{R_{1} a} d a
$$

where the ages are normalized with respect to the classical generation time $T$ of (4).

\subsection{Two case studies}

For the majority of stable populations, one observes close agreement between the actual net maternity function $\phi(a)$ and the lognormal model net maternity function $\varphi(a)$ given in 3.4.1. Typically, there is a slight discrepancy in the most fertile age range, where the model overestimates the fertility. This overestimate is compensated by an underestimate elsewhere. The two case studies exhibited in this section use the data tabulated in [9], which are based on 5-year age classes.

\subsubsection{Malaysia}

Figure 2 compares the actual net maternity function $\phi(a)$ for peninsular Malaysia in 1985 (solid lines, points + ) with the lognormal model function $\varphi(a)$ that the formula (15) provides (dotted lines, points o). The numerical parameters $R_{1}=0.0642$ per annum, $t=$ 27.81 years, and $u=0.215$ are computed from the data in [9] using respective "binned" or discretized versions of (8), (9), and (10). For the 1985 Malaysian population, the classical generation time (4) was $T=28.48$ years (see Table 1 below). As noted in 3.3 , the logarithmic generation time $t=27.81$ years is less than the classical generation time.

\subsubsection{USA}

The discrepancy between the reality and the lognormal model, as an overestimate at the fertile ages and corresponding underestimate elsewhere, becomes more pronounced for the USA population in 1985. The significance of such discrepancies is discussed in 4.1 below.

The actual and lognormal model net maternity functions for the USA in 1985 are illustrated by Figure 3, which uses the same conventions as Figure 2, and is obtained the same way. The numerical parameters for this case are $R_{1}=0.0354$ per annum, $t=24.34$ years, and $u=0.230$. The classical generation time was $T=26.39$ years. 


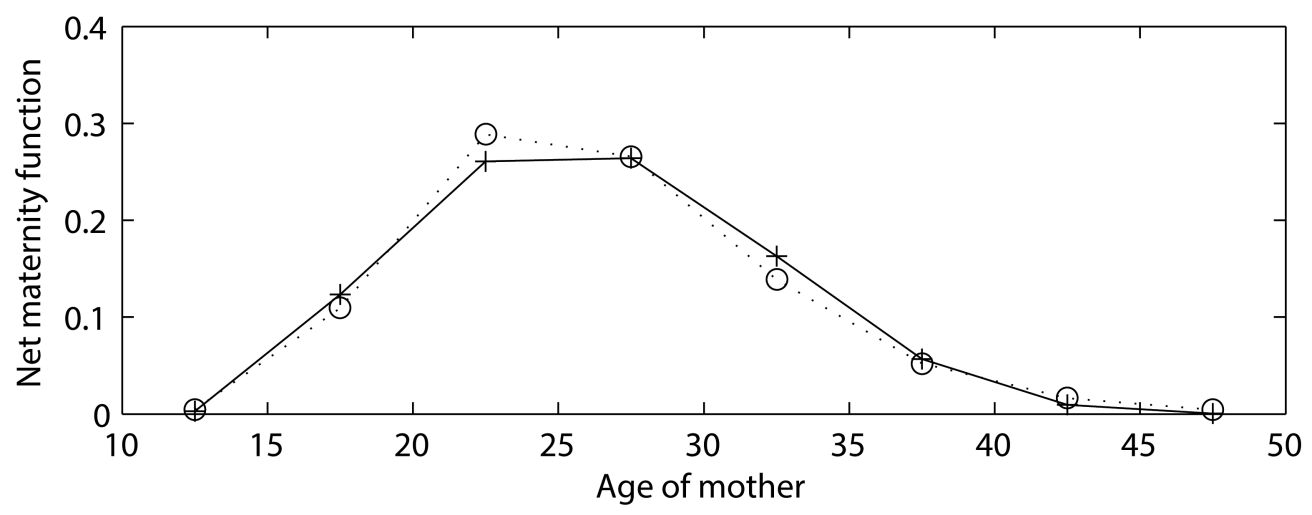

Figure 3: Net maternity functions for USA, 1985. Solid: actual $\phi(a)$. Dotted: the lognormal model $\varphi(a)$.

\subsection{Beyond the lognormal model}

If discrepancies between a model and the reality are to be diminished or eliminated (to within the accuracy of the experimental observations), a more exact model than the lognormal could be derived by imposing additional constraints, beyond (12), (13), and (14), in the information-theoretic entropy maximization procedure of 3.4. This provides a topic for future research, and will not be discussed further here.

\section{Using the model}

This section discusses three interrelated applications of the lognormal parametrization of the net maternity function: diagnostics, prediction, and long-term tracking.

\subsection{Immediate diagnostics}

As observed at the beginning of 3.5, most historic populations present a close agreement between the actual net maternity function $\phi(a)$ and the lognormal model net maternity function $\varphi(a)$ of 3.4.1, comparable to that seen in the 1985 peninsular Malaysian population in Figure 2. Comparison between the two functions, revealing potential significant divergences, offers a diagnostic procedure for detecting various influences that may be acting within the population. This comparison, which only involves the data for one particular time period, may be described as immediate diagnostics.

\subsubsection{Diagnosing 1985 USA}

The divergence between the two functions observed within the 1985 USA population is displayed in Figure 3. The actual fertility for the 20-25-year-old age class falls noticeably short of what the model predicts. This discrepancy may be indicative of families postponing childbirth while younger potential mothers were focusing on their immediate career goals. In the 15-20-year-old age class, the excess of actual fertility relative to the model may reflect the United States' notoriously high teenage birth rate in the nineteen-eighties, the "highest in the developed world" [16].

\subsection{Predicting future states}

Taken together, the triple $\left(R_{1}, t, u\right)$ of parameters forms a vector which may be used for the prediction of future states of a population. This technique is illustrated by Table 1, which displays the vector for the peninsular Malaysian population (based on [9]) for five-year 
intervals from 1970 to 1985 . The individual components for 1970-1980 were extrapolated quadratically to obtain estimated components for 1985, as shown on the table. These components compare closely to the true components for 1985, the largest error being a $6 \%$ underestimate for the deviation. Figure 4 then compares the lognormal model net maternity function determined by these estimated parameters (dotted lines, points o) with the actual 1985 net maternity function (solid lines, points + ). Of course, the agreement is not as close as that observed in Figure 2.

\begin{tabular}{c|ccc|c} 
& $R_{1}(/ \mathrm{yr})$. & $t(\mathrm{yrs})$. & $u$ & $T$ (yrs. $)$ \\
\hline True 1970 & 0.0781 & 27.09 & 0.2361 & 27.83 \\
True 1975 & 0.0692 & 27.04 & 0.2331 & 27.55 \\
True 1980 & 0.0642 & 27.24 & 0.2220 & 27.75 \\
\hline True 1985 & 0.0642 & 27.81 & 0.2150 & 28.48 \\
\hline Est. 1985 & 0.0630 & 27.69 & 0.2022 & \\
\hline Error & $-2 \%$ & $-1 \%$ & $-6 \%$ & \\
\hline
\end{tabular}

Table 1: Parameter vectors and generation times for peninsular Malaysia.

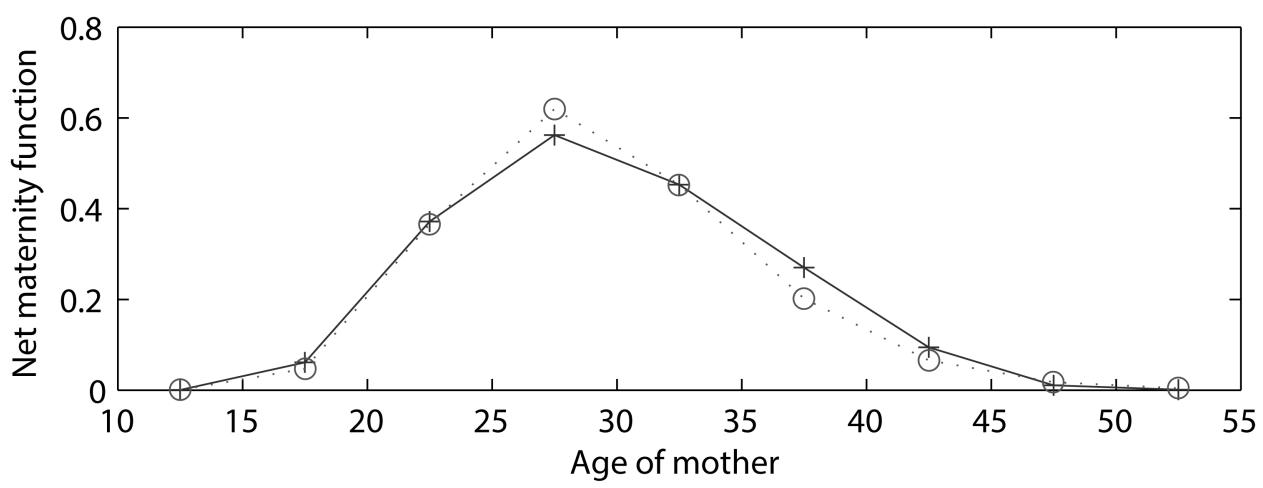

Figure 4: Net maternity functions for Malaysia, 1985. Solid: actual $\phi(a)$. Dotted: the lognormal model $\varphi(a)$ projected from 1970-1980 (compare 4.2).

\subsubsection{Predictive diagnostics}

Just as the discrepancy between the the real net maternity function and the lognormal model net maternity function at a given time period formed the basis for the immediate diagnostics of 4.1, so the kind of discrepancy observed in Figure 4, between the actual net maternity function and a projected lognormal model net maternity function based on previous parameter values, may also serve as the basis for a predictive diagnostic procedure. For example, using Figure 4 to diagnose the 1985 Malaysian population predictively, one observes a shift in fertility from the 25-30-year-old age class to the 35-45-year-old age classes. This shift gives a more detailed picture of the effect that is observed in the increased 
generation times (both classical and logarithmic) of the 1985 population, as displayed in Table 1.

\subsection{Long-term tracking}

Using five-year age classes, the sheer number of distinct discrete net maternity function values (5) at a given point, e.g., 9 for Malaysia or 8 for the USA in 1985 (Figures 2-3), makes it hard to use these values for comparison of the state of a population at differing stages. On the other hand, the parametrization (15) of the net maternity function allows one to analyze the entire history of a population by tracking the parameters over time. The following section examines a notably well-documented example, that of Sweden.

\subsection{The Swedish dataset}

Among the demographic data tabulated by Keyfitz and Flieger [8, 9] for specific human populations at varying times over the preceding centuries, the most complete set is for Sweden. Figure 5 shows the record of the logarithmic generation time $t$ (in years) and deviation $u$ for this population over 5 -year steps from 1780 to 1985 . Recall that the deviation $u$ is dimensionless. To compare $t$ and $u$ for common display, the latter has been multiplied by a proportionality factor $k$ (with the dimension of time). The specific choice of 102 years as the value for $k$ is discussed in 4.4 .4 below.

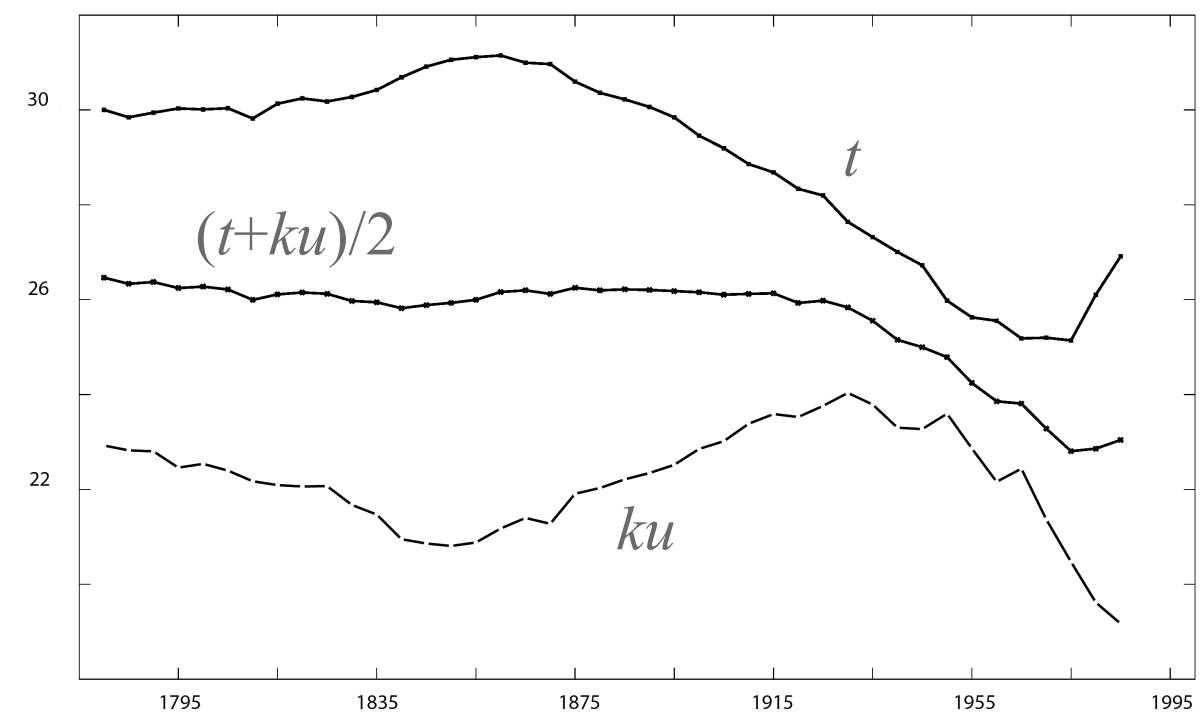

Figure 5: Tracking the parameters $t$ and $u$ for the Swedish population over the 1780-1985 time period. The upper curve depicts the logarithmic generation time $t$ (in years). This quantity reaches its maximum at around 1860 and then starts to decrease as industrialization sets in. The lower curve depicts the dimensionless deviation parameter $u$ multiplied by the proportionality factor $k$ (set at a value of 102 years). The intermediate curve records the average $(t+k u) / 2$, which remains remarkably constant (to within demographic accuracy) from the beginning in 1780 to the onset of the modern era around 1930 .

\subsubsection{The demographic transition}

The logarithmic generation time $t$ shows a general increase up to 1860, but then declines steadily until 1965. The demographic transition measured by $t$ in the 1860-1870 time period coincides with the onset of industrialization: 
In the case of Sweden, however, it seems reasonable to use the term "industrial transformation" to define the period from the mid-nineteenth century until the First World War. Here, we can establish with certainty both an accelerating average growth rate, a substantial increase in the industrial sector's share of the economy, and important organisational changes in the industrial sector. [10]

A more precise time point, correlating with the maximum of the logarithmic generation time $t$, is determined by the initial imports of railway locomotives to Sweden in 1856 $1872[5]$.

\subsubsection{Comparing the generation times}

Overall, the logarithmic generation time $t$ behaves in similar fashion to the classical generation time $T$, but appears to smooth out some of the more random fluctuations of the latter.

\subsubsection{Duality between $t$ and $u$}

In general, as $t$ increases, $u$ decreases, and vice versa. Given an upper age limit on fertility of around 45-55 years, this relationship is indeed to be expected: Increasing the mean in the normal distribution forces the variance to decrease.

\subsubsection{A conservation principle}

Nevertheless, an unexpectedly tighter relationship between $t$ and $u$, a form of conservation principle, is observed from 1780 until the onset of the modern era around 1930: The existence of a value for the proportionality factor $k$ such that $t+k u$ is approximately constant. The particular $k$-value used in Figure 5 (namely 102 years) was obtained by minimizing the variation of $t+k u$ over the time points from 1780 to 1925 .

\subsubsection{Dimension reduction}

In effect, the conservation principle means that two parameters alone, the renormalization constant $R_{1}$ and the logarithmic generation time $t$, are sufficient to determine the logarithmic model net maternity function over time periods within the validity of the principle. Specifically, the experimental relation

$$
\frac{t+102 u}{2}=26
$$

observed from Figure 5 allows one to substitute $u=\left(\begin{array}{ll}52 & t\end{array}\right) / 102$ in (15).

Note that while in theory one might equally choose $R_{1}$ and $u$ as the two basic parameters within the purview of the conservation principle, the resultant substitution $t=52 \quad 102 u$ would be less stable with respect to errors in the value of $u$ and the experimental determination of the constant 26 years via Figure 5 .

\section{Conclusions}

While the net maternity function does not lend itself well to modeling in terms of universal time, it has been parametrized effectively as a function of the logarithm of a mother's age. Choice of this independent variable leads to dimensionless quantities that do not have to be rescaled when the units of time are changed. The parametrized lognormal model provides a close fit to the real function for stable populations, while divergences between the model and real function may serve as a diagnostic for new influences on a population. Reduction of the specification of the net maternity function to a small number of parameters enables those parameters to be tracked in long-term studies of a population, and also serves as the 
basis of techniques for predicting future states of a population. Among the parameters, the logarithmic generation time emerges as an important population variable.

\section{References}

[1] B. J. Andresen, S. Shiner, and D. E. Uhliger, 2002. Allometric scaling and maximum efficiency in physiological eigen time. Proc. Nat. Acad. Sci. U.S.A., 99, 5822-5824.

[2] G. Backman, 1940. Lebensdauer und Entwicklung. Arch. für Entwicklungsmechanik, 140, 90-123.

[3] E. L. Crow and K. Shimizu, 1968. Lognormal Distributions. New York, NY: Marcel Dekker.

[4] P. Harremoës and F. Topsøe, 2001. Maximum entropy fundamentals. Entropy, 3, 191226 (electronic journal).

[5] R. L. Hills, 1997. Beyer Peacock Locomotive Order List. London: British Overseas Railways Historical Trust.

[6] N. Keyfitz, 1968. Introduction to the Mathematics of Population. Reading, MA: Addison-Wesley.

[7] N. Keyfitz, 1985. Applied Mathematical Demography (2nd. ed.). New York, NY: Springer.

[8] N. Keyfitz and W. Flieger, 1968. World Population: An Analysis of Vital Data. Chicago, IL: University of Chicago Press.

[9] N. Keyfitz and W. Flieger, 1990. World Population Growth and Aging. Chicago, IL: University of Chicago Press.

[10] L. Magnusson, 2000. An Economic History of Sweden. London: Routledge.

[11] E. L. O'Neill, 1963. Introduction to Statistical Optics. Reading, MA: Addison-Wesley.

[12] I. Prigogine, 1947. Etude Thermodynamique des Phénomènes. Paris: Dunod.

[13] K. Schmidt-Nielsen, 1984. Why is Animal Size so Important? Cambridge: Cambridge University Press.

[14] C.E. Shannon, 1948. A mathematical theory of communication. Bell System Tech. J. 27, 623-656.

[15] J. D. H. Smith, 2001. Some observations on the concepts of information-theoretic entropy and randomness. Entropy 3, 1-11 (electronic journal).

[16] UNICEF, 2001. A league table of teenage births in rich nations. Innocenti Report Card No. 3, July 2001. Florence: UNICEF Innocenti Research Centre. 